\title{
PEDAGOGIE PAR PROJET: UNE ÉTUDE DE CAS \\ DANS L'ENSEIGNEMENT SUPERIEUR TECHNOLOGIQUE
}

\begin{abstract}
RÉSUMÉ: Cet article rapporte les résultats d'un questionnaire proposé aux étudiants d'une formation d'enseignement supérieur technologique en génie électrique et informatique industrielle. L'objectif de ce questionnaire est d'évaluer le profit que les étudiants ont retiré d'un enseignement des techniques de gestion de projet, et l'utilisation qu'ils en ont fait, d'une part au cours d'une série de travaux pratiques utilisant la pédagogie par projet, d'autre part au cours de leur stage de fin d'études en entreprise.

Mots-clés: Pédagogie par Projet. Gestion de Projet. Études et Réalisations. Stages en Entreprises. Électronique et Informatique Industrielle.

\section{BASED TEACHING- PROJECT: A CASE STUDY ON THE VOCATIONAL ROUTE IN THE UNIVERSITY}

ABSTRACT: This article gives an account of the results of a questionnaire to be filled in by students of electrical engineering and industrial data processing who were in a higher training course in their vocational route. The objective is to evaluate the benefit they got from an instruction in project management techniques, together with the use made of these techniques, both during a series of practical activities based on a projectbased teaching, and during their final placement in companies. Keywords: Project-based Teaching. Project Management, Engineering Design and Prototype Development. Placements in companies. Electronics and Industrial Data Processing.
\end{abstract}

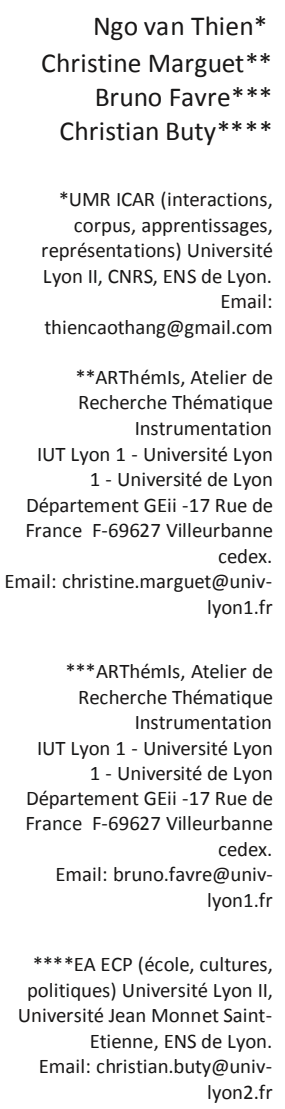

****EA ECP (école, cultures, politiques) Université Lyon II, Université Jean Monnet SaintEtienne, ENS de Lyon. Email: christian.buty@univIyon2.fr 


\section{INTRODUCTION}

L'intérêt pour la pédagogie par projet s'est manifesté depuis longtemps dans l'enseignement supérieur. On peut attribuer cet intérêt à deux raisons principales: d'une part les compétences des étudiants, qui sont suffisamment développées pour mener des travaux de longue durée dans des contextes où ils sont plus autonomes et où la réponse n'est pas connue à l'avance par l'enseignant; d'autre part l'objectif de professionnalisation de l'enseignement supérieur, car la réalisation d'un projet se rapproche du type de tâche demandé dans le milieu professionnel, et fait une transition entre les activités proprement scolaire et les stages que les étudiants doivent réaliser en entreprise à l'issue de leur formation.

Une des caractéristiques essentielles des projets est leur durée, relativement grande, plus grande en tous cas que les activités pédagogiques habituelles. Cette durée impose une cohérence dans l'action et dans la réflexion, un suivi organisationnel de l'activité. Les projets sont donc une opportunité pour les étudiants de développer des méta-compétences relatives à l'organisation de leur travail, qu'ils ont assez peu d'occasions d'exercer dans les autres activités sur le lieu de leur formation universitaire. Pour les enseignants aussi, le positionnement par rapport au savoir et aux étudiants, la nature des interactions qu'ils ont avec eux, les tâches qu'ils doivent assurer et les outils qu’ils sont amenés à utiliser sont transformés par ce type de pédagogie.

Le présent texte constitue la première partie d'une recherche en cours sur la pédagogie par projet, basée sur une étude de cas dans un département d'IUT (GEii: génie électrique et informatique industrielle) de l'Université de Lyon I. Il associe dans son élaboration deux chercheurs en didactique des sciences et deux enseignants qui ont construit l'enseignement étudié (ils sont par ailleurs des chercheurs dans le domaine de l'électronique dédiée à l'instrumentation). Il rend compte d'une évaluation d'une innovation pédagogique, portant sur un dispositif de réalisation de projet existant depuis plusieurs années. Il est important de préciser que les chercheurs en didactique des sciences qui cosignent cette contribution n'ont participé ni à la mise en place du dispositif, ni à la définition de l'innovation qui a été évaluée par le questionnaire dont nous allons parler. Ils ont donc ici un positionnement clinique, dépourvu d'ingénierie.

\section{CADRE THEORIQUE, LA PEDAGOGIE DE PROJET}

La pédagogie de projet fait partie de ce qu'on appelle classiquement les pédagogies actives (voir par exemple Gauthier et Tardif, 2005); elle renvoie à un modèle d'apprentissage que nous allons caractériser, et qui selon (Abdallah, 2009) est lié aux modèles cognitivistes, constructivistes, et socio-cognitivistes. 


\section{Définition de la notion de projet}

À partir des apports d'auteurs tels que (Boutinet, 1990), (Tilmal, 2004), (Francoeur-Bellavance, 1995), (Huber, 2005) et (Proulx, 2004) on peut définir un projet pédagogique comme l'ensemble des activités de résolution d'un problème issu de la vie réelle. Ces activités mettent en œuvre une démarche d'anticipation complète, et se déroulent dans un temps déterminé; elles nécessitent l'élaboration d'un planning. Leur résultat est double: d'une part une production concrète, matérielle ou intellectuelle; d'autre part le développement chez l'apprenant de savoirs de haut niveau ainsi que de compétences transversales.

\section{Caractéristiques et apports de la pédagogie par projet}

La littérature met en avant un certain nombre de caractéristiques des pédagogies de projet, et leurs effets favorables sur les apprentissages.

- Un projet doit mettre l'apprenant dans une situation qui constitue un défi (Abdallah, 2009); il est initié à partir d'une thématique concrète de la vie (Lebrun, 2007); il doit s'orienter vers une production concrète et évaluable (Perrenoud, 2002; Proulx, 2004; Abdallah, 2009).

- L'enseignant a un rôle de guide, de motivateur et de médiateur. L'apprenant est actif et responsable dans l'apprentissage (Raby, Beaudry, \& Viola, 2007; Proulx, 2004; Dumas, 2002).

- L'apprentissage par projet est marqué par la collaboration, la coopération, la coordination (Raby, Beaudry, \& Viola, 2007).

- L'évaluation se compose de plusieurs phases qui prennent place à différentes étapes du déroulement du projet et non pas seulement à l'étape terminale (Bordallo \& Ginestet, 1993 ; Proulx, 2004 ; Tilman, 2004; Boutinet, 1990).

- L'apprentissage par projet développe des savoirs, des savoir-faire, des savoir-être et des savoirs s'effectuant dans l'action, des savoir-devenir (Huber, 2005 ; Mahieu, 1992; Roegiers \& Ketele, 2001); il développe également des compétences transversales (Le Boterf, 2003; Lafortune, 2009; Proulx, 2004; Raby, Beaudry, \& Viola, 2007), renforce l'autonomie et la responsabilité, développe la confiance en soi chez l'apprenant (Proulx, 2004, Huber, 2005). Il favorise le travail en équipe (Perrenoud, 2002; Colet, 2002 ; Mahieu, 1992); il permet la mise en application de l'interdisciplinarité (Bordallo \& Ginestet, 1993; Abdallah, 2009 ; Valzan, 2003).

\section{Les phases d'un projet}

Comme il a été dit, un projet se déroule dans le temps suivant plusieurs phases, qu'on peut décrire comme suit. 


\section{Une phase de préparation:}

L'enseignant doit pouvoir proposer un large choix de sujets de projet, pour lesquels il clarifie et explicite ses intentions pédagogiques. Il doit également permettre une répartition de ces sujets entre les apprenants selon un processus démocratique (Proulx, 2004; Forrest, 2011). Enfin il remet pour chaque projet une ébauche de synoptique. Les apprenants effectuent l'analyse du besoin, l'étude de faisabilité et établissent le cahier des charges (Bordallo \& Ginestet, 1993).

\section{Une phase de structuration du projet:}

Un projet doit d'abord être décomposé en étapes, puis chaque étape découpée en tâches (Abdallah, 2009). Celles-ci doivent ensuite être planifiées (Proulx, 2004; Tilman, 2004). Différents outils tels que l'organigramme des tâches, les diagrammes de Gantt, ou de Pert sont alors utilisés (Bordallo \& Ginestet, 1993).

\section{Une phase de réalisation du projet:}

Réaliser un projet, c'est le lancer dans la réalité. C'est une période longue pour laquelle il est nécessaire de confronter la réalisation des tâches aux prévisions du planning de référence. A ce propos, (Boutinet, 1990) parle de gestion des écarts. (Forrest, 2011) formule ainsi l'enjeu de cette gestion des écarts: « quelle régulation, quels ajustements apporter, comment garantir la cohérence entre la mise en œuvre et les objectifs » (p.38)

\section{Une phase d'évaluation du projet:}

L'évaluation se compose de plusieurs moments qui prennent place à différentes phases du déroulement du projet et non pas seulement à la phase terminale. Selon (Proulx, 2004; Forrest, 2011) l'évaluation formative est fréquente et immédiate en cours de projet. L'évaluation sommative est effectuée à travers l'évaluation de produits finaux.

\section{CONTEXTE DE L'ÉTUDE}

\section{La formation IUT - GEii:}

Cette étude s'appuie sur l'observation de plusieurs modules d'enseignement dispensés dans le département GEii de l'IUT Lyon1 (France).

Les IUT (Instituts Universitaires de Technologies) ont depuis leur création en 1966 l'objectif de former en deux ans des Techniciens Supérieurs. Le département GEii de l'IUT de Lyon I dispense un enseignement dans le domaine du Génie Electrique et de l'Informatique Industrielle. La formation se fait en accord avec un Programme Pédagogique National (PPN) qui organise 1800 heures encadrées de cours, TD, TP, (trois semestres S1 à S3 de 510 heures chacun et un semestre 
S4 de 270 heures) et un stage de fin d'études (semestre S4) de 10 semaines en entreprise. Les enseignements sont structurés en modules "cœur de compétences" et en modules "complémentaires" permettant à l'étudiant d'adapter son parcours en fonction de son projet personnel et professionnel (approfondissement technologique, renforcement des compétences professionnelles et ouverture scientifique). Des parcours type sont proposés, avec spécialisation en électronique, ou électrotechnique, ou automatique et informatique industrielle. Tous les étudiants ont dans leur parcours une suite de trois modules ER (Études et Réalisations), deux en semestre S3 et un en S4. Cette suite ne constitue en fait qu'un seul "projet ER" de 74 heures conduit tout au long de la 2eme année.

\section{Le projet "Études et Réalisations":}

Vu du côté des étudiants, le projet ER consiste à concevoir et réaliser un ensemble opérationnel, appareil complet ou bien carte électronique, dans le domaine de l'électronique, l'électrotechnique, les automatismes, l'informatique industrielle, les réseaux, le traitement du signal.

Vu du côté enseignant, le but est en outre de placer chaque étudiant dans un contexte aussi proche que possible de celui qu'il trouvera en milieu professionnel, dès son stage en entreprise.

Ainsi, chaque étudiant se voit confier un sujet à réaliser en binôme durant sa 2 ème année. Il gère son déroulement (planning, conception, achats, fabrication, essais, validations, rapports...) en autonomie. Il est encadré par un enseignant issu du monde industriel ou scientifique (un enseignant pour une douzaine d'étudiants) dont le rôle correspond à celui de l'ingénieur "chef de projet" en entreprise.

\section{Les sujets:}

Les sujets sont:

- proposés par les enseignants en fonction de leurs propres thèmes d'intérêt personnels et du parcours des étudiants.

- très variés.

- souvent innovants ; ils peuvent aussi se faire en collaboration avec des laboratoires ou des industriels. L’enseignant n'a donc pas une solution a priori.

- tous différents; ainsi, en 2011-2012, il y avait 8 groupes d'environ 24 étudiants en 2 ème année, ce qui correspond à 96 sujets différents proposés par 12 intervenants. Ceci est un choix du département étudié, il ne découle pas obligatoirement du PPN.

- Nous donnons ci-dessous quelques exemples de réalisations traitées :

- Véhicules: véhicule électrique alimenté par énergie solaire, instruments pour véhicule participant à la course internationale Shell Eco-Marathon, $\ldots$ 
- Instruments de mesure et test: synthétiseur numérique de fréquence, boussole électronique, mesure des polluants atmosphériques par carte d'acquisition autonome, journal lumineux, acquisition GPS, station météo, transmission de données sans fil...

- Moto: éléments de tableau de bord, dépressiomètre pour synchronisation des carburateurs, ...

- Musique: métronome avec affichage de la pulsation, sampler, accordeur guitare, ...

- Concours robot (entre les départements GEii de France): suivi de ligne par infrarouge, suivi de ligne par caméra vidéo, ...

- Recherche médicale: unités d'acquisition et de mesure en IRM, échographie...

\section{Les particularités de l'enseignement ER:}

L'enseignement des projets ER est donc sensiblement différent des cours, TD, TP, même s'il fait bien sûr appel aux connaissances acquises. Il demande en outre de la part de l'étudiant des investigations personnelles, permet d'acquérir une méthode de travail, et d'aborder directement les problèmes liés au cahier des charges, au choix des méthodes et des solutions, aux contraintes liées aux fournisseurs (prix et délais), aux échéanciers et éventuels contretemps. De plus, le cahier des charges de départ peut être parfaitement défini, ou bien évoluer en fonction de certains critères (prix de revient, durée de réalisation, extension ou restriction des caractéristiques souhaitées ou permises...). Le rôle de l'enseignant est alors surtout de valider ou non les solutions proposées par l'étudiant, de l'aider à faire ses choix, de le guider sur de nouvelles voies si nécessaire, et éventuellement d'adapter la complexité de la réalisation effective en fonction des compétences de la paire d'étudiants. Il faut noter ici que tous les étudiants n'ont pas les mêmes enseignants, et que les pratiques de ceux-ci à propos de la gestion de projet constituent une variable peu maîtrisée.

Une des caractéristiques essentielles du projet ER est que l'étudiant travaille la plupart du temps en autonomie. Il dispose pour cela des équipements nécessaires pour la réalisation de son projet:

- de postes informatiques: recherche documentaire, internet, $\mathrm{CAO}$ (schémas, simulation, placement, routage), développement (C, $\mathrm{C}++$, Matlab, LabVIEW, ...), bureautique, composants programmables...

- d'équipements de réalisation de circuits imprimés, traversants ou CMS

- de postes de perçage

- de postes de mesure et test (un par binôme), équipés de générateur de signaux, oscilloscope, multimètre, alimentations stabilisées, poste de soudage... 
- d'instruments spécifiques: oscilloscopes HF à mémoire, générateurs HF, alimentations de puissance...

De plus, les techniciens du pôle technique du département GEii peuvent ponctuellement intervenir sur des réalisations délicates ou complexes.

\section{Les évaluations ER:}

Chaque étudiant est guidé, mais aussi évalué au cours et en fin de sa réalisation, au moyen de grilles communes pour l'ensemble des groupes de la promotion. On appréciera notamment:

- ses aptitudes professionnelles (méthode, organisation, autonomie, efficacité, connaissances, jugement, imagination...)

- son comportement général (dynamisme, sociabilité, conscience professionnelle, clarté...)

Il doit aussi fournir au long de l'année des éléments de suivi et d'évaluation:

- un rapport de pré-étude, incluant cahier des charges, organigramme des tâches, planning, synoptique de la réalisation (à la séance 5 environ)

- un état d'avancement des travaux (soutenance orale, séance 10)

- un rapport écrit de conception (séance 14)

- le produit fini et sa documentation technique (séance 25).

Chacun de ces éléments doit être présenté sous la forme la plus professionnelle et valorisante possible, en se rapprochant des façons de procéder utilisées dans le monde industriel que l'étudiant rencontrera lors de son stage ou dans la vie active.

\section{La formation à la gestion de projet}

En 2008, le nouveau Programme Pédagogique National du DUT « Génie Electrique et Informatique Industrielle » préconisait la mise en place de 10 modules complémentaires. A cette occasion, le département GEii de l'université de Lyon I a décidé de laisser en libre choix aux étudiants, deux de ces modules parmi les 8 proposés. A été alors créé un module «Management de projet». En effet, de plus en plus d'entreprises utilisent les techniques de gestion de projet. Elles y sont souvent amenées au moment de la mise en place d'une démarche qualité, la gestion de projet faisant partie des éléments incontournables de cette démarche. Les étudiants y seront donc confrontés soit dès la fin de leur 2ème année d'IUT au moment du stage en entreprise, soit dans leur vie professionnelle en tant que membre d'une équipe projet, ou en tant que chef de projet. Cependant, la mise en place de ce module ne répond pas totalement à la nécessité de former tous les étudiants aux notions de base de la gestion de projet, puisqu'il n'est accessible qu'à 26 étudiants. La solution choisie par l'équipe pédagogique a été de mettre en place $2 \mathrm{~h} 30$ de travaux dirigés, au démarrage des projets ER, c'est-à-dire au début du semestre 3. En effet, pour que le projet joue son rôle pédagogique il 
faut qu’il soit bien mené. Cet enseignement s'appelle « Introduction à la gestion de projet ». Cette introduction présente l'objectif de la gestion de projet (conduire et mener à bien son projet), les acteurs du projet (maittre d'ouvrage, maittre d'œuvre) et leur contrat (le cahier des charges). Est abordée ensuite la notion de tâche, tout d'abord sous forme d'une liste des tâches à accomplir pour réaliser le projet électronique ; puis il leur est demandé de structurer leur décomposition en tâches élémentaires à l'aide de l'organigramme des tâches. Puis leur est présenté un outil de planification: le diagramme de Gantt. Leur est enfin présentée une modélisation pour les différentes phases d'un projet et les documents qui y sont associés, en établissant le lien avec le déroulement de leur projet ER.

Dans la mesure où tous les étudiants sont formés à ces techniques de base, le champ d'action du module complémentaire au choix « Management de projet» a été élargi aux différents rôles du chef de projet et à la technique de conduite de réunion.

\section{PROBLEMATIQUE}

Compte tenu du dispositif d'enseignement des méthodes de gestion de projet qui a été décrit plus haut, les enseignants ressentaient la nécessité d'une part d'une enquête de satisfaction auprès des étudiants sur les contenus proposés dans l'innovation pédagogique, en vue de son amélioration, et d'autre part d'évaluer l'utilisabilité de ces contenus soit en interne (pour la conduite du projet ER) soit en externe (lors des stages en entreprise). Puisque l'enseignement à la gestion de projet s'adresse à deux populations différentes, à tous les étudiants dans un premier temps (« introduction à la gestion de projet » en début d'année, deux cents étudiants environ), et aux seuls étudiants (une vingtaine) qui ont choisi le module « management de projet » au cours de l'année, les questions qu'on peut se fixer pour cette recherche se regroupent en trois grandes catégories :

- dans quelle mesure les TD d'introduction à la gestion de projet sont-ils perçus par les étudiants comme une aide à la réalisation des projets ER (influence interne à l'IUT)? Quels sont les retours des étudiants sur la forme et le contenu de ces TD?

- en quoi les éléments fournis lors de ces TD ont-ils été utilisés lors du stage en entreprise (influence externe à l'IUT)?

- quel retour les étudiants donnent-ils sur le module «management de projet » ? En quoi les outils conceptuels et méthodologiques fournis ontils été utilisés pendant leur stage?

- Le mode le plus naturel de collection de données pour répondre à ces questions, étant donné le nombre d'étudiants concernés, est l'administration d'un questionnaire qui va être présenté ci-dessous (il est fourni en annexe). 


\section{LA COLLECTE DES DONNEES: LE QUESTIONNAIRE}

Nous avons choisi de proposer ce questionnaire anonyme aux étudiants, en fin de $2^{\text {ìme }}$ année, au moment des soutenances de stage, pour les raisons suivantes:

- Le projet ER est terminé pour tous les étudiants; on peut donc recueillir leur avis sur la façon dont il s'est déroulé.

- Comme ces soutenances concernent l'ensemble de la promotion, le document de l'enquête a pu être remis en main propre à une fraction importante des étudiants (par l'intermédiaire des jurys de soutenance) et, pour la plupart, ils ont eu le temps de le remplir et de le rendre immédiatement. Cela a permis d'assurer un taux de réponse satisfaisant (112/160, soit $70 \%$ de la promotion).

- cela donne un sens aux 2ème et 3ème volets de cette enquête portant sur la gestion de projet vécue en entreprise ainsi que sur le module « management de projet » pour les étudiants qui l'ont suivi.

Dans la première partie du questionnaire, nous souhaitions avoir le sentiment des étudiants sur l'utilité de ces TD comme aide à la gestion de leur projet ER. De plus, en vue d'une évolution éventuelle de ces TD, nous nous posions les questions suivantes: le volume d'heure est-il adéquat? Faut-il envisager des interventions de l'enseignant de TD au cours des premières séances de projet comme soutien à la rédaction du cahier des charges et à la réalisation de l'OT et du planning? Les étudiants souhaitent-ils plus de soutien des enseignants de projet sur ces aspects? Faut-il les impliquer davantage?

Dans la deuxième partie, nous souhaitions analyser la présence de techniques de gestion de projet en entreprise et leur utilisation par les étudiants stagiaires. L'utilisation de ces techniques est-elle corrélée à la taille de l'entreprise? Les étudiants stagiaires ont-ils été impliqués par leur tuteur dans cet aspect gestion de projet? Ont-ils spontanément pensé à utiliser ces techniques, ce qui marquerait une appropriation de leur part?

La troisième partie portait plus spécifiquement sur le module complémentaire « Management de projet », et ne pouvait donc concerner que les deux douzaines d'étudiants qui avaient suivi cet enseignement. Nous souhaitions recueillir l'impression globale de ces étudiants vis-à-vis de ce module complémentaire et savoir s'ils avaient été confrontés à l'aspect « conduite de réunion » pendant leur stage en entreprise.

En définitive, nous avons recueilli 112 réponses, parmi lesquelles 16 étudiants avaient suivi le module complémentaire «management de projet ». Dans la suite, les résultats seront donnés en pourcentages ; ces pourcentages porteront sur les 112 réponses, sauf précision contraire. 


\section{METHODE DE TRAITEMENT DES DONNEES}

Le questionnaire est constitué pour l'essentiel de questions fermées où on donnait aux étudiants le choix entre quelques modalités. Deux espaces d'expression libre avaient été ménagés, mais ils ont été très peu utilisés et ils ne seront pas analysés ici de façon systématique. Chaque questionnaire rempli par les étudiants a été affecté d'un numéro d'ordre, commençant par son numéro de groupe, suivi d'un numéro personnel (exemple 403-25, pour le $25^{\text {ème }}$ questionnaire du groupe 403). Le codage d'une question particulière consiste dans la plupart des cas à cocher les modalités présentes dans le questionnaire, suivant les réponses de l'étudiant; pour chaque question, une modalité "non réponse » a cependant été rajoutée. Cela signifie que les 13 questions de la première partie ont 5 modalités, sauf la quatrième, qui demandait un volume horaire indicatif; pour cette quatrième question, on a codé sur 6 modalités, adaptées aux réponses observées: $0 \mathrm{~h}, 1 \mathrm{~h}, 2 \mathrm{~h}$, $3 \mathrm{~h}, 4 \mathrm{~h}$, non réponse. Les 10 questions de la deuxième partie, portant sur le stage en entreprise, étaient à réponses binaires (oui/non), sauf la seconde qui demandait la taille de l'entreprise; on a donc codé sur trois modalités (oui/non/non réponse), et sur quatre modalités pour la $2^{\text {ème }}$ question (moins de 20 personnes/de 20 à 100 personnes/plus de 100 personnes/non réponse). La troisième partie, réservée aux étudiants qui avaient suivi le module optionnel de management de projet, contenait 9 questions à quatre réponses possibles, qui ont donc été codées sur cinq modalités.

La première façon, la plus immédiate, de traiter ce questionnaire, est de faire un tri à plat des réponses aux différentes questions, pour effectuer un simple dénombrement de réponses.

Dans un deuxième temps, nous avons cherché des corrélations entre les réponses à différentes questions. Ces corrélations permettent soit de préciser la validité de certaines estimations fournies par les tris à plat, soit de faire apparaitre des relations entre certains aspects de l'investigation. Techniquement, les corrélations seront établies par des tris croisés entre réponses.

On peut également sélectionner un sous-échantillon, sur la base des réponses à une question particulière; on peut par exemple traiter à part les étudiants qui ont effectué leur stage dans une entreprise de moins de 20 personnes. Précisons maintenant quels tris croisés nous avons effectués pour obtenir des corrélations entre réponses, qui fassent sens au regard de nos questions de recherche. Une première série de remarques peut porter sur le stage en entreprise pour l'ensemble de la promotion. On peut d'abord se demander si la taille de l'entreprise est une facteur important pour l'application durant le stage des techniques de gestion de projet, en totalité ou en partie. La réponse passe par une corrélation recherchée entre les réponses à la question 2.2 et les questions $2.1,2.3,2.6$. On peut chercher s'il y a des différences significatives entre la population générale et la sous- 
population qui a suivi le module "management de projet », en cherchant également pour cette sous-population si la taille de l'entreprise influence la réponse aux questions 3.4 à 3.8 .

Une deuxième direction de réflexion peut porter sur le rapport qu'il y a entre intérêt pris aux enseignements qui ont été proposés et estimation par l'étudiant de l'usage qu'il fera de ces méthodes de travail à l'avenir; ce sont alors des corrélations entre les questions 1.1 et 3.1 d'une part, 1.2, 2.9, 3.3 et 3.9 d'autre part, qui sont recherchées.

Toutes ces statistiques descriptives ont été réalisées grâce au logiciel Modalisaํํ․

\section{RESULTATS}

\section{Le sentiment des étudiants sur l'introduction à la gestion de projet}

Dans l'ensemble, les étudiants ont considéré comme positive l'introduction à la gestion de projet qui leur a été dispensée en ouverture des séances de TP ER: 82 $\%$ des répondants indiquent « oui » ou « oui un peu » à la première question. Très logiquement, $82 \%$ également estiment (en regroupant encore les réponses « oui » ou « oui un peu », ce que nous ferons dans la suite, sauf avis contraire) que l'horaire imparti était suffisant (question 1.3); par conséquent la question 1.4, demandant d'indiquer un horaire souhaitable, devient sans grand intérêt.

\section{L'utilité de la gestion de projet dans les TP ER}

Si on considère maintenant l'intérêt de cette introduction à la gestion de projet non plus généralement, mais sous l'angle de son applicabilité aux TP ER que les étudiants ont réalisés à sa suite, le taux de satisfaction reste élevé, à un niveau de 70 \% (question 1.2). Le rôle de cette introduction est donc perçu comme positif, sans être tout à fait primordial, ce que montrent les questions 1.5 et $1.6:$ sur les $70 \%$ d'étudiants qui estiment que leur projet ER a abouti, $38 \%$ déclarent que c'est « grâce aux outils et à l'organisation proposés dans » cette introduction.

Les trois questions 1.7, 1.8 et 1.9 visaient à explorer les limites de l'adhésion des étudiants aux outils qui leur avaient été proposés, de façon plus détaillée que la question 1.2. Elles mentionnaient explicitement les deux dispositifs principalement exposés dans l'introduction, c'est-à-dire l'organigramme des tâches et le planning; elles étaient formulées de façon à favoriser l'expression de problèmes éventuels. $72 \%$ des étudiants estiment (question 1.7) que faire un organigramme et un planning N'EST PAS « une perte de temps » (regroupement des réponses « plutôt pas » et « non »). La question 1.8 introduisait une nuance supplémentaire, en mentionnant la maîtrise par l'étudiant de ces outils, évidemment en construction lors du TP ER, comme cause possible de perte de temps; $53 \%$ des étudiants estiment ne pas avoir perdu de temps ou peu ; 
cependant $37 \%$ répondent « oui un peu », ce qui indique qu'ils reconnaissent avoir eu besoin d'un temps d'apprentissage.

Les questions suivantes (1.10 à 1.12bis) allaient un peu plus loin encore, en introduisant une référence à un besoin éventuellement ressenti par les étudiants, d'aide pour l'application des outils à leur projet particulier, soit de la part de l'enseignante qui a dispensé l'introduction à la gestion de projet, soit de la part de l'enseignant qui a encadré le projet ER proprement dit. Les réponses sont rassemblées dans le tableau 1 ci-dessous.

Tableau 1: pourcentages des étudiants qui estiment qu'une aide leur aurait été utile (réponses " oui » et « oui un peu »).

\begin{tabular}{|l|l|l|}
\hline $\begin{array}{l}\text { Point sur lequel une aide } \\
\text { aurait été utile }\end{array}$ & $\begin{array}{l}\text { De la part de l'enseignante du } \\
\text { TD introductif }\end{array}$ & $\begin{array}{l}\text { De la part de l'enseignant de } \\
\text { TP ER }\end{array}$ \\
\hline $\begin{array}{l}\text { Elaboration du cahier des } \\
\text { charges }\end{array}$ & $44 \%$ & $58 \%$ \\
\hline $\begin{array}{l}\text { Découpage en tâches } \\
\text { élémentaires }\end{array}$ & $45 \%$ & $55 \%$ \\
\hline Elaboration du planning & $36 \%$ & $43 \%$ \\
\hline
\end{tabular}

Pour autant que les différences qu'on observe dans ce tableau soient significatives, ces pourcentages peuvent s'interpréter de deux façons:

- les étudiants attendent un peu plus d'aide de la part de l'enseignant qui encadre leur projet, plutôt que de l'enseignante qui a présenté le TD introductif, et qui évidemment n'est pas présente pendant les TP ER.

- suivant le sujet, la demande d'aide n'est pas la même : l'élaboration du planning semble plus facile que la décomposition en tâches élémentaires, et surtout que l'élaboration du cahier des charges.

Pour répondre à cette difficulté exprimée, une modification a été apportée au contenu de l'introduction à la gestion de projet, l'année qui a suivi ce questionnaire: les étudiants ont dû rédiger, pendant cette introduction, le cahier des charges d'un mini-projet d'électronique qu'ils avaient tous réalisé au semestre 2. De cette façon, l'enseignement répondait également à une critique implicite des étudiants, apparente dans la réponse à la question 1.13 (56\% des étudiants auraient souhaité un exemple plus technique que celui qui avait été utilisé dans la première version de cette introduction, l'organisation d'une soirée étudiante).

\section{L'utilisation de ces notions dans le stage en entreprise}

La question d'applicabilité de cette formation dans la vie professionnelle des étudiants, telle qu'ils peuvent l'entrevoir à travers leur stage de fin d'études, était pertinente, puisque selon leur perception, $70 \%$ des étudiants se sont trouvés dans 
des entreprises appliquant des techniques de gestion de projets (question 2.1). La question 2.2 en donne une explication partielle: $43 \%$ des étudiants sont allés dans une grande entreprise (plus de 100 personnes); $26 \%$ sont allés dans une entreprise de taille moyenne, comprise entre 20 et 100 personnes. Or on peut penser que ces deux types d'entreprises ont une plus grande probabilité d'employer des techniques de gestion de projet identifiables.

Un tri croisé entre les questions 2.1 et 2.2, d'ailleurs, fournit une précision intéressante: une plus forte proportion d'étudiants $(80 \%$ ) qui ont fait leur stage dans une moyenne entreprise ont identifié qu'elle recourait à des techniques de gestion de projet, en comparaison de ceux qui ont fait leur stage dans une grande entreprise $(70 \%)$. Ce dernier pourcentage n'est pas très différent de celui qui concerne les petites entreprises: 66,7\% (mais sur un effectif plus restreint, 18 étudiants).

Cependant, que l'entreprise où ils ont fait leur stage applique des techniques de gestion de projet ne signifie pas que les étudiants en stage eux-mêmes les ont systématiquement appliquées. Si on analyse en effet les réponses aux questions 2.3 à 2.7, qui précisaient les outils de gestion de projet effectivement utilisés par les étudiants, on obtient le tableau 2 ci-dessous. Ce tableau, qui regroupe les questions 2.4 et 2.5 , et qui intègre le résultat de tris croisés avec la taille de l'entreprise, donne le pourcentage de réponses positives dans chaque catégorie d'entreprises.

Tableau 2: répartition des réponses positives aux questions en fonction de la taille de l'entreprise de stage.

Les pourcentages sont rapportés aux nombres d'étudiants qui ont fait leur stage dans les différents types d'entreprises. La dernière ligne toutefois ne concerne qu'une souspopulation de l'échantillon, les étudiants qui ont répondu oui à la ligne précédente

\begin{tabular}{|l|l|l|l|l|}
\hline Question posée & $\begin{array}{l}\text { Stage en petite } \\
\text { entreprise }\end{array}$ & $\begin{array}{l}\text { Stage en } \\
\text { moyenne } \\
\text { entreprise }\end{array}$ & $\begin{array}{l}\text { Stage en grande } \\
\text { entreprise }\end{array}$ & $\begin{array}{l}\text { Totalité des } \\
\text { stages }\end{array}$ \\
\hline $\begin{array}{l}\text { Avez-vous rédigé } \\
\text { un cahier des } \\
\text { charges? }\end{array}$ & $33 \%$ & $10 \%$ & $27 \%$ & $22 \%$ \\
\hline $\begin{array}{l}\text { Avez-vous suivi } \\
\text { un planning? }\end{array}$ & $28 \%$ & $52 \%$ & $37 \%$ & $41 \%$ \\
\hline $\begin{array}{l}\text { Avez-vous } \\
\text { spontanément } \\
\text { rédigé ce } \\
\text { planning? }\end{array}$ & $60 \%$ & $27 \%$ & $56 \%$ & $48 \%$ \\
\hline
\end{tabular}

On constate donc qu'une fraction seulement des étudiants qui disent que leur entreprise utilise des techniques de gestion de projet, les ont effectivement utilisées par eux-mêmes. Les écarts sont particulièrement nets pour les entreprises moyennes, puisque $80 \%$ des étudiants estiment que l'entreprise se sert de 
techniques de gestion de projet, alors que $10 \%$ d'entre eux ont pu rédiger le cahier des charges de leur stage, et que $52 \%$ seulement ont suivi un planning. De plus (voir la ligne 3 du tableau 2), sur ces $52 \%$ qui ont suivi un planning, seuls un quart environ l'ont rédigé eux-mêmes (une proportion deux fois plus petite que dans les petites ou les grandes entreprises). On peut avancer l'hypothèse que le stagiaire dans une moyenne entreprise a tendance à être moins autonome vis à vis de son encadrement. On peut aussi, à l'inverse, émettre l'hypothèse que les tuteurs en entreprise ne s'attendent pas à ce que les étudiants de GEii soient capables d'utiliser ces techniques; de fait, elles n'apparaitront explicitement que dans le nouveau programme national, qui entrera en vigueur en septembre 2013.

Ces résultats décrivent en quelque sorte les pratiques relatives aux techniques de gestion de projet qui ont eu cours pendant le stage de fin d'études des étudiants qui ont répondu. Les trois questions suivantes (2.8 à 2.10) donnent une idée de la représentation que les étudiants se sont faite de l'utilité pour leur stage des techniques de gestion de projet.

En premier lieu, $83 \%$ des étudiants estiment que leur stage a abouti, est une réussite $^{2}$ (question 2.8). Mais 35\% seulement estiment que les techniques de gestion de projet ont contribué à cette réussite (question 2.9). C'est assez normal, compte tenu de ce que nous avons dit sur l'écart entre constater l'emploi dans l'entreprise de techniques de gestion de projet, et les employer soi-même pour son stage. Au final néanmoins, $88 \%$ des étudiants - la quasi totalité - affirme que l'expérience du stage les a convaincu de l'intérêt de s'organiser pour mener à bien un projet (question 2.10).

\section{Le cas des étudiants ayant suivi le module optionnel « management de projet ॥}

Rappelons que dans cette dernière partie seuls 16 étudiants ont répondu ('effectif du module était de 24 étudiants); les pourcentages qui sont donnés dans ce paragraphe portent sur ces 16 réponses.

Sur le module complémentaire, son contenu, les avis sont très positifs: $81 \%$ des étudiants se disent satisfaits de ce module (question 3.1) ; 81\% disent avoir appris quelque chose (question 3.2); $87 \%$ sont décidés à utiliser ces techniques par euxmêmes (question 3.3).

En ce qui concerne le stage de fin d'études de cette population particulière d'étudiants, $81 \%$ d'entre eux ont décomposé leur sujet de stage en tâches élémentaires (question 3.4). Par contre seuls $50 \%$ d'entre eux ont participé à des réunions d'avancement de projet (question 3.5); il faut prendre en considération qu'ils n'en étaient pas maitres, car c'est la pratique de l'entreprise qui s'impose, soit qu'il ne fasse pas partie de la culture d'entreprise d'organiser des telles réunions, soit que les stagiaires n'y participent pas. Mais quand ils y ont participé, ils ont repéré les types de conduite de réunion tels que l'enseignement du module les avaient présentés (question 3.7): leur enseignement a pu être mis en pratique. Enfin, une minorité d'entre eux a effectué une présentation lors de ces réunions 
(25\%, question 3.8); et encore faut-il tenir compte du fait que souvent les entreprises font répéter en interne les soutenances terminales des étudiants, ce qui ne rentrait pas totalement dans le cadre de la question mais a pu être confondu par certains étudiants.

La dernière question (3.9) se termine en forme de plébiscite: $87 \%$ des étudiants estiment que ce module leur sera utile dans leur vie professionnelle. Très logiquement, un tri croisé avec la question 3.1 indique que plus les étudiants ont été satisfaits du contenu du module, plus ils considèrent qu'il leur sera utile professionnellement.

\section{CONCLUSION}

Il apparait, au vu de cet ensemble de données et des résultats que nous en avons dérivés, que le dispositif d'explicitation des outils de gestion de projet, qui a été mis en œuvre en préambule des TP ER dans ce département GEii d'IUT, constitue une aide réelle pour les étudiants, à la fois en interne, pour le déroulement des TP ER, et en externe, pour le stage de fin d'études en entreprise. En tous cas, c'est la représentation que les étudiants en ont, puisque ce sont leurs déclarations qui sont ici prises en compte. Ils portent un jugement positif non seulement sur le contenu de ce qui leur a été exposé, mais aussi sur l'usage qu'ils ont pu en faire. On a vu cependant que cet usage est différencié suivant plusieurs dimensions:

- les outils techniques de gestion de projet: le cahier des charges paraît plus difficile que le planning dans l'application au TP ER; cette hypothèse est corroborée par le fait qu'en stage, l'élaboration d'un cahier des charges est moins souvent requise du stagiaire que le suivi ou l'élaboration d'un planning, parce que le cahier des charges estsouvent imposé par l'entreprise.

- La situation dans laquelle l'étudiant se trouve: tous les étudiants ne sont pas invités à utiliser de façon active les outils de gestion de projets pendant leur stage, et ce de façon différente suivant la taille de l'entreprise. Il est bien évident que l'objectif de l'encadrement du stage dans l'entreprise n'est pas d'améliorer les compétences de l'étudiant sur les techniques de gestion de projet; il est avant tout que l'objectif du stage soit atteint.

On peut ajouter, dans le même sens, que suivant le groupe de TP ER où l'étudiant va se trouver, l'enseignant qui encadre le groupe accordera une importance variable aux techniques de gestion de projet, et par conséquent l'intérêt que l'étudiant leur portera sera également variable.

Nous avons rapporté ici une étude exploratoire, basée sur un questionnaire pour recueillir des informations à l'échelle de toute une promotion d'étudiants. La 


\section{Ngo van Thien | Christine Marguet | Bruno Favre | Christian Buty}

contrepartie de cette étendue est bien entendu le caractère assez général des affirmations que l'on peut énoncer sur la façon dont les étudiants ont utilisé ces techniques de gestion de projet dans les deux contextes dont nous avons parlé. Notre étude se poursuit par l'analyse fine de l'activité en TP ER de deux étudiants, tout au long des 24 séances de cet enseignement. Nous décrirons ainsi comment les outils de gestion de projet ont été mis au service de la réussite de ce projet particulier, avec un grain nettement plus fin.

\section{BILLETS}

${ }^{1}$ Modalisa.com

2 Un tri croisé permet d'émettre l'hypothèse que la réussite du stage dépend légèrement de la taille de l'entreprise (78\% pour les petites entreprises, $83 \%$ pour les moyennes, $94 \%$ pour les grandes).

\section{REFERENCES BIBLIOGRAPHIQUES}

ABDALLAH, F. . Meta-modélisation pour décrire et instrumenter une situation d'apprentissage de pédagogie par projet collectif. Thèse de doctorat en informatique. Le Mans: Université du Maine, 2009.

BORDALLO, I., \& Ginestet, J.-P. . Pour une pédagogie du projet. Paris: Hachette-Education, 1993.

BOUTINET, J.-P. Anthropologie du projet. Paris: PUF, 1990.

COLET, N. R. Enseignement universitaire et interdisciplinarité: Un cadre pour analyser, agir et évaluer.

Bruxelles: De Boeck Supérieur, 2002.

DUMAS, B. Les rôles de l'enseignant en pédagogie de projet. Québec français, 126, 64-66, 2002.

FORREST, C. (dir.) La pratique de la pédagogie par projet (2e éd.). Alma: Axone, 2011.

FRANCOEUR-BELLAVANCE, S. Le travail en projet. Québec français, 97, 42-45, 1995.

GAUTHIER, C., \& Tardif, M. (dir.). La pédagogie: théories et pratiques de l'Antiquité à nos jours (2e éd.). Montréal: G. Morin, 2005.

HUBER, M. Apprendre en projets: la pédagogie du projet-élèves (2e éd.). Lyon: Chronique Sociale, 2005.

LAFORTUNE, L. La pédagogie du projet et développement des compétences transversales : changement de posture pédagogique. Education Canada, 49(5), 16-20, 2009.

LE BOTERF, G. Compétence et navigation professionnelle. Paris: Éditions d'Organisation, 2000.

LEBRUN, M. Théories et méthodes pédagogiques pour enseigner et apprendre: Quelle place pour les TIC dans l'éducation? (2e éd.). Bruxelles: De Boeck Supérieur, 2007.

MAHIEU, P. Travailler en équipe. Paris: Hachette, 1992.

PERRENOUD, P. Apprendre à l'école à travers des projets : pourquoi ? Educateur, 14, 6-11, 2002.

PROULX, J. L'apprentissage par projet. Sainte-Foy: PUQ, 2004.

RABY, C., BEAUDRY, N., \& VIOLA, S. (dir.). Modèles d'enseignement et théories d'apprentissage: de la pratique à la théorie. Anjou: Éditions CEC, 2007.

ROEGIERS, X., \& KETELE, J.-M. D. Une pédagogie de l'intégration: Compétences et intégration des acquis dans l'enseignement (2e éd.). Bruxelles: De Boeck Supérieur, 2001.

TILMAN, F. Penser le projet: concepts et outils d'une pédagogie émancipatrice. Lyon: Chronique sociale, 2004. VALZAN, A. Interdisciplinarité et situations d'apprentissage. Paris: Hachette éducation, 2008. 
Pedagogie par Projet : Une Étude de Cas Dans L'enseignement Superieur Technologique

Data Recebimento: 10/10/2012

Data de Aprovação: 13/11/2012

Data Versão Final: 11/01/2013 